\title{
Spinal Meningioma: Pathophysiology, Diagnosis and Management
}

\author{
Muhammad Imran Qadir $^{1 *}$ and Anam Shahzadi ${ }^{2}$ \\ ${ }^{1}$ Institute of Molecular Biology \& Biotechnology, Bahauddin Zakariya University, Multan, Pakistan \\ ${ }^{2}$ Faculty of Pharmaceutical Sciences, Government College University, Faisalabad, Pakistan
}

Received: 眥 May 22, 2018; Published: 盋 May 29, 2018

*Corresponding author: Muhammad Imran Qadir, Institute of Molecular Biology \& Biotechnology, Bahauddin Zakariya University, Multan, Pakistan

\begin{abstract}
Tumors have several types that can build up in or near the spine. There are many types of spinal tumors. They can appoint the spinal cord, nerve roots or the vertebrae (bones of the spine) and pelvis. Spinal tumors have two classes. A spinal tumor can be primary, meaning it comes from cells within or near the spine. Primary tumors of the spine are uncommon. More commonly a spinal tumor that is found is a secondary spinal tumor. This means that the tumor traveled there from someplace else in the body. Tumors can be benign (non-cancerous) or malignant (cancerous). Arise from the arachnid "cap" cell of arachnid villi. Meningiomas are slow growing usually present next to dura matter and the 2nd most common tumors of CNS and primary in nature. They increased intracranial pressure when it enters sometimes in the skull.
\end{abstract}

\section{Introduction}

The cervical part of the spinal cord contains 7 vertebras which starts from the end of skull and attach to the uppermost point of thoracic spine. The thoracic part contains 12 vertebrae and lies in the chest region .this region are different from others because here ribs attached to the vertebrae. It ends when it joins with lumbar vertebrae. The lumbar spine has 5 vertebrae and it joins with sacrum or pelivis at the edge. Vertebral body causes the formation of vertebrae by a round black of bones where the bony ring attaches to it and when these bony rings join with each other they form neural arch which is a hollow tube. In this hollow tube spinal cord is placed which confined by the bone. It starts from the end of medulla or brain stem and ends to the1st 2 nd lumbar spine where it is called cauda equine because of having the shape of heresies tail $[1,2]$.

\section{Structure of Spinal Cord}

Spinal cord surrounds by cerebro spinal fluid. It is hollow in the middle it function is to communicate the messages to and from the brain and also body. All vertebras join with each other in vertical position and from spinal column where spinal cord is lie. Spinal column keeps the body straight. Spinal cord has three layers the outermost is thick layer called dura matter. Then due to the similarity in structure with spider web. The middle one is arachnold matter and innermost is thinner called pia matter. Due to those layers tumors spread in the spinal cord and causes the spinal Meningioma [3].

\section{Angioblastic Meningiomas}

Meningiomas are classified as angioblestic because it contain many blood vessels of different sizes. conexity because unlike groups lies in the anterior portion of rolandic fissure of the sulici of brain psammonatus bodies [4].

\section{Symptoms of Meningioma}

Vary with the location this means that spinal cord and nerve root is compressed. Symptoms may nuisance be rapidly or slowly onset meningioma itself not alters you by plain in spinal cord at night may alters you by its presence. nuisance, Seizures, Hearing, reminiscence loss, Arm or leg failing, visualization changes (fuzzy or dual vision), queasiness or sickness, Loss of consciousness, backside pain, Pain in the arms, legs or chest from compacted nerves etc are the common symptoms of meningioma [5]. 


\section{Causes of the Spinal Meningioma}

A primary spinal tumor runs from one generation to another. It rises within the cord or it's nearby it cells. It arise in the never roots, pelvis, spinal cord or spinal boys. The primary tumors contains a number of abnormal genes but nobody known about it canse. It becomes more complicated when it compressed the spine. It censed paralysis and loss of blander and making us unstable by damage the vertebral canal which assist the cord [6].

\section{A Secondary Spinal Tumor}

Is more common these are cancerous in nature and traveled from one part to another part of the body. They cause skin cancer, lung cancer, breast, prostate, kidney andthyrod gland etc. In these days Meningiomas become a serious problem after therapy failure. So endocrine therapy is advised. Molecular mechanism can help to diagnose and prognose the disease. Hormonal manipulation also helpful for diagnosis this diseases.

Molecular mechains not only helpful the diagnosis but it also helps in the treatment of disease $[7,8]$. Types of Spinal Meningioma Tumors of spinal cord are three types. Intramedudlary tumors, intradural extramedullary and extramedullary turners. intramedullary lies inside the cord they damage the spinal cord. The 2nd one are located inside the dura but outside the spinal cord (dura is a hard fibrous sheat that cover the cord). it compress the spinal cord the 3rd one lies with the vertebrae and outside the cord. It can damage cord dura and also vertebrae. Another type which lies in $b / w$ the intramedullary or extramedullary spaces is pediatric spinal cord, the extramedullary is of two types. They are intradural or extradural areas [9]. The intradural include nerve sheath tumors e.g. meningiomas. The extradural contains nueroblastomas, sarcomas and other primary areas. Tumors may be cancerous or non-cancerous. The most common type is (meningioma or schwanommas) are non-concerous and extramural are cancerous [10]. Meningioma contains 1.2 percent spinal compartment as compared to add other meningiomas. meningioma mostly is intradural and it is proved by latest technologies like neuroimaging techniques and micro neurosurgical producers. Now this meningioma can be removed easily. Meningioma mostly occur is older but its presence in younger is due to a genetrical disorder known neurofibromatosis 2 or related to aggressive historical subtypes [11]. Local pain is the symptom of spinal meningioma but it cannot be diagnosed until it shows neurological damages or deformation. Lesions appear ventrally on the cord which disturbs the neurological skills of patient. Meningiomas found 25 percent of all spinal tumors and intradural in nature. but it can be treating in best manner by surgery and proved by $75 \%$ to $85 \%$ operated patients. Many authors have studied about its surgery and division of lesions Out of 25\%, 2-4 \% is primary spinal cord tumours of CNS. Meningiomas caused by arachnoidal cells and most of them near to the venous sinuses which is the site of its formation. It is just like a clone shape lying on the dura. It covers on the space and grey [12].
Meningioma is very common and arises from meningial coverings of brain and coral. They arise from arachinodal cap cells but their based cannot be proven yet. Their treatment surgery and radiation therapy shows good results, The first treatment is surgery which goal to finish the tumour but the method of treatment adopted by studying the patient's history, signs and the effects of surgery on it [13].

When the tumour progressed the radiation therapy is given but it a non-typical method which is still controversial. Sometime after radiation therapy again the growth of tumour occurred. This is due to the bad functional prognosis to improve the outputs. Ionizing radiation is also a source of treatment. The chances of meningioma occurrence increased due to mismatch of healthy gene with poor one or deletion of Nuerofibromatosis type 2 genes (NF2) which cause cancerous tumour changing in the NF2 causes the occurance of more than half meningiomas. This is an autosomal disorder which causes mutation in NF2 on chromosome 22q [14] After the classification of tumours the surgery becomes easy and radiation therapy shows better results. This proves a better way of elder patient. Hyperostosis and endomosis are frequently occurred in meningioma cases problems of bone also occurred in patients. Gradually bones of spine destroyed. According to location of tumour in spinal cord the patients are categorized as sever, moderate and mild. The role of metalloproteinase (MMP) and its level can be determined by gelatin zymography, reverse transcription quantitative PCR analysis and immunohistochemical analysis [12]. Extramedullary intradural are rare $15 \%$ to all the tumours in the body are spinal tumours. (50-50\%) are extradural, $40-45 \%$ are intradural $5 \%$ are intramedullary and $40 \%$ are extramedullary. All these produce pain.

\section{Diagnosis of Spinal Meningioma}

For diagnostic purposes different method are adopted [13]. For diagnosis the doctor will ask the questions about signs and aur feelings and how this disease effect our daily life works. The doctor ask about our past life that we have been diagnosed any tumour in past. Then doctor takes our physical; test by check our walking style then different laboratory works adopt to diagnose the cancer.

\section{Magnetic Resonance Imaging (MRI)}

In this procedure magnetic waves are used to see the soft tissues of body in the cord and nerves. The dye also used which is given intravenously. The areas are diagnosed in parts.

\section{Computed Tomography (CT)}

This method is adopted when MRI not available. The dye is injected in the spinal fluid so different parts are identified. Myelogram is the method of insertion of dye.

X-Rays

This method is used to only see the changing in the structure of bone. In primary cancer changing's in the structure of bone. In 
primary cancer changing's in the bone not occurred. Bone scans are used to see the tumours and fractures. Sometimes different elements like technetium (Tc) are used by inserting in the vein. It becomes viscous at the site of cancer. Now MRI mostly used for the diagnosis of this disease. It provides exact location of different types of tumours by detecting the size and shape of lesions. Metastasis, meningiomas and schwannomas are categorized as intradural tumours. Schwannomas and neurofibromas are dumb-bell like shape $[14,15]$. Osteopontin protein also taking role in the diagnosis of spinal meningioma.

\section{Osteopontin protein}

It is the first discovered in 1986 in osteoblasts and it SBLING glycoprotein. Its is also called as sialoprotein I(BSP-1) and secreted phospho protein 1 it is a gene product and safe in the coming species; It is known as linking protein as its name shows "Osteo" that it appeared in the bone and "Pomtin" from "Pons" so it acts as a bridge and also found in various tissues. The other names of it are sialoprotein I and $44 \mathrm{~K}$ BPP (Bone phospho protein). It is an organic part of bone and extra cellular structural protein. It is found on the long arm of chromosome 4 [16].

\section{Relationship of Osteopontin Protein And Meningi- oma Diagnosis}

It is very help full in meningioma diagnosis because it is the basic structure of bone so its lack causes the diagnosis of disease. It is mostly found in blodder, prostate and brain tumours and the other cancers like skin revealed low OPN concentration. It is an integrin-binding protein; it has a prominant relationship with tumours. Its concentration and size is also related with special cancer places like bladder, colon, and kidney. Larynx, mouth and salivary glands, this protein is involved in the formation of tumours and it is distributed in different body sites. A hard relation b/w this protein and different tumors [17].

\section{Role of Osteopontin Protein in Cancer and Inflam- matory Disease}

It is occurred at different tumours sites including lung cancer, breast cancer, stomach cancer and mesothelioma and meningiomas. The level of OPN at different body sites helpful for the diagnosis and treatment of the diseases like osteoporosis, cancer metastasis and antoimmune disease $[17,18]$. To study the OPN level scientists studied 350 human tumours and 113 normal tissues at different body areas. Tumour included tissues from breast, colon, kidney, lung, skin, brain and liver etc [19].

\section{Treatments of Spinal Meningioma}

After through study about patient's disease, signs and imaging studies the treatment is allowed. Then treatment consists of different methods like surgical and non-surgical. Sometimes waves or chemotheraphy is adopted by the help of oncologist (If a doctor specializes in cancer). Before treatment diagnosis is necessary if this cannot be done then patient has to face a forever problem [20].
Non-Surgical Treatment: All the tumors cannot be damage by surgery. Sometimes Non-surgical method is adopted after seeing the important signs of disease.

Medication: Medications are also used for the treatment of tumours. They cut off the supply of blood to tumours hence their growth is diminished or they reduced the swelling of the cord which causes in the reduction of compression on the nerves, vertebrae and spinal cord. These medications sometimes used temporary or forever according to the patient condition.e.g corticosteroids. Analgesica are also used e.g. narcotics. In osteoporosis, biophosphonates are used because of the destruction in the bony area of the spine has been occurred [21].

Embolization: In this method blood vessels are burned and blood supply to the cancer is cut-off. Most of the meningiomas are treated by this.

Chemotherapy: This procedure is used in the case of malignant or cancerous tumours. In the case of secondary tumour when it is traveled to reach another area is used [22].

Radiation Therapy: This is also used to remove the cancer without surgery.

\section{Surgical Method}

This method is adopted for primary tumours or benign or noncancerous tumours. Most of the tumours are treating that they cannot cause neurological problems. Neurological disorders can be diminished by using new methods. In these methods we used ultrasonic aspirators, microsurgery. In ultrasonic aspirators we used the waves of sound to remove the cancer. It also converts the meningioma into pieces. In microsurgery microscop is used to view the full area and to survive any damage from the surrounding areas [23].

Endocrine Therapy: In the cases of PGR-positive meningioma endocrine therapy is used. By the treatment of surgery and radio therapy a number of patients again affected by meningioma. Then these patients have to face different problems. After the success in the treatment of breast cancer this hormone manipulating method is adopted to treat the meningioma. So this method of treatment is adopted for those patients who have meningioma after radiotherapy or surgery [24].

Mife Pristoner: It is taken only for the treatment of PGRpositive meningioma. It minimized the transcriptional activity of PGR-complex. For this purpose it adopts different mechanisms of concentrations. It is antagonistics with palliation of meningioma.

Tamoxifen: It decreases DNA production and estrogen effects at the site of cancer. It is also taken orally and also known as ER Modullator. Patients have not important change in the production of tumors [25]. 


\section{Conclusion}

Spinal Meningioma is a very common but a dangerous disease. There are lot of researches has done on this disease and this struggle is continue. I selected this disease because I want to study the brief explanation about the symptoms, treatments, diagnosis, medications, classification, discoveries and different theories of scientists on this disease. I have studied many review articles and made this assignment. Basically spinal tumors have two common types Tumors can be benign (non-cancerous) or malignant (cancerous). Therapies are very useful for the treatment of spinal meningioma and different therapies perform a useful role for the rehabilitation of meningioma patients e.g. radio therapy. Spinal meningioma may be primary or secondary meningioma. It may be extramedullary or intramedullary. Primary spinal cord tumors are anatomically separable into two broad categories: intradural intramedullary and intradural extramedullary. Osteopontin protein alson plays a very important role in the identification of spinal meningioma. Other methods also adopt for its identification and treatment. Surgical and non-surgical method used for its treatment. Non-surgical methods contains Medications, Embolization, Chemotherapy, Radiation therapy.

\section{References}

1. Vijayakumar Javalkar, Bharat Guthikonda, Prasad Vannemreddy, Anil Nanda (2009) Increased prevalence of obesity and obesity related postoperative complications in male patients with meningioma. Journal of publication of neurological society of India 57(6): 772-776.

2. Jain, Sharma, Sarkar, Suri, Garg, et al. (2009) Association of malignant brain tumors and cancers of other sites, Journal of cancer research and therapeutics 5(4): 324-327

3. Roger, Turbin, Kathryn Pokorny (2004) Diagnosis and Treatment of Orbital Optic Nerve Sheath Meningioma. cancer journal 11(5): 334-341.

4. Bracard, Abdel Kerim, Thriller, Klein, Anxionnat, et al. (2010) Nutritional factors in the etiology of brain tumors: potential role of nitrosamines. Journal of mindscape today (1): 47.

5. Changhong, Naiyin, Yuehuan, Lianzhong (2009) journal of Korean neurosurgical society.

6. Sibony, Krauss, Kennerdell (2005) Primary brain tumors as second primary: a novel association between meningioma and colorectal cancer. Journal of neuro opthalmology (25): 2 .
7. Nam Hee Kim, Seung Yeob Yang, Joon-Bum Koo, Sang Wuk Jeong (2009) Occipital Neuralgia as the Only Presenting Symptom of Foramen Magnum Meningioma. journal of clinical neurology 5(4): 198-200.

8. H Hakan Oruckaptan, Figen Soylemezoglu, Tezer Kutluk, Nejat Akalan (2000) journal of pediatric neurosurgery 32(5).

9. Chen, fag, Southern medical journal 102(11): 1164-1166.

10. (2010) Recurrences of meningioma: predictive value of pathological Features and hormonal growth factors. Journal of Turkish Nuerosergury 20(2): 241-246.

11. Nadir sunumu, Lateral Kafa kaidesi Primer Miksom (2010) Journal of Turkish neurosergury 20(1): 86-89-12.

12. Bagdasar Arseni, Carol Darila, Adela maagdalena bioban, Teodora cameelia coman, Dtto n JJ, et al. (2009) Ramanian Journal of morphology and embroyology 50(4): 713-717.

13. Karp, Zimmerman, Borit A, Spencer W (1974) primary intraorbital meningiomas. Arch opthalmol 91: 24-28.

14. Boyne, Gree St Lonis, Mo (2002) Mosby Ultrasound of spinal Cord.

15. Cyrus chargari, Lionel vedrine (2008) Dliver Banducean and nioclas magne endocrine related cancer 15: 931-941.

16. Aghi, Eskandar, Carter, Curry, Barker (2007) Hormonal manipulation of meningiomas. Endocrinology journals (61): 754-760.

17. Journal of clinical oncology (13): 2931-2935.

18. National Journal of Neuroscience (112): 463-470.

19. Kaplan, Novikov, Modan (1997) Nutritional factors in the etiololgy of spinal tumors. American Journal of emiclieniology 146: 832-841.

20. Baser, Evans, Gurman (2003) Neurofibromatosis. Current opinion in neurology (16): 27-33.

21. Edward, Leonhardt, Grass Handel, Journal of the society for Gynecology Investigation (7): 22-24.

22. Gazzeri, Galarza M anel Gazzerig, New England Journal of medicince (357): 2411-2412.

23. Verheigen, sprong, Jacobs, Donker, Role of antiprogestational therapy for meningiomas. Journal of Caner (37): 1488-1495.

24. Wahiab, Al Azzawi (2003) spinal Meningioma and hormonal influence, clinical endoriroinology 6(4): 285-292.

25. Grundbrory, Weiss Reisell, Spitz, Ahmadi, An evaluation of tamoxifen in unresectable or refractory meningiomas, Journal of steroid Biochemestry and Molecular Biology (56): 93-98.

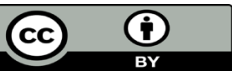

This work is licensed under Creative Commons Attribution 4.0 License

To Submit Your Article Click Here: Submit Article DOI: 10.32474/OAJOM.2018.01.000121

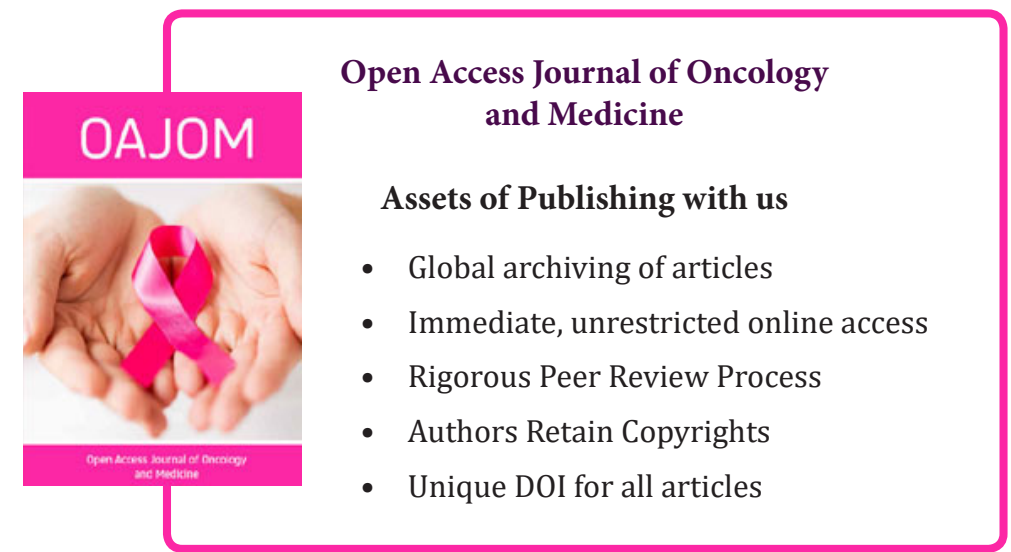

